\title{
DOSSIER
}

\section{ECONOMÍA SOCIAL Y SOLIDARIA EN MOVIMIENTO: DIÁLOGOS Y APORTES DESDE UNA PSICOLOGÍA SOCIAL PLURAL}

SOCIAL AND SOLIDARITY ECONOMYIN MOTION: DIALOGUES AND CONTRIBUTIONS FROM A PLURAL SOCIAL PSYCHOLOGY

$$
\text { NATANIA TOMMASINO? }
$$

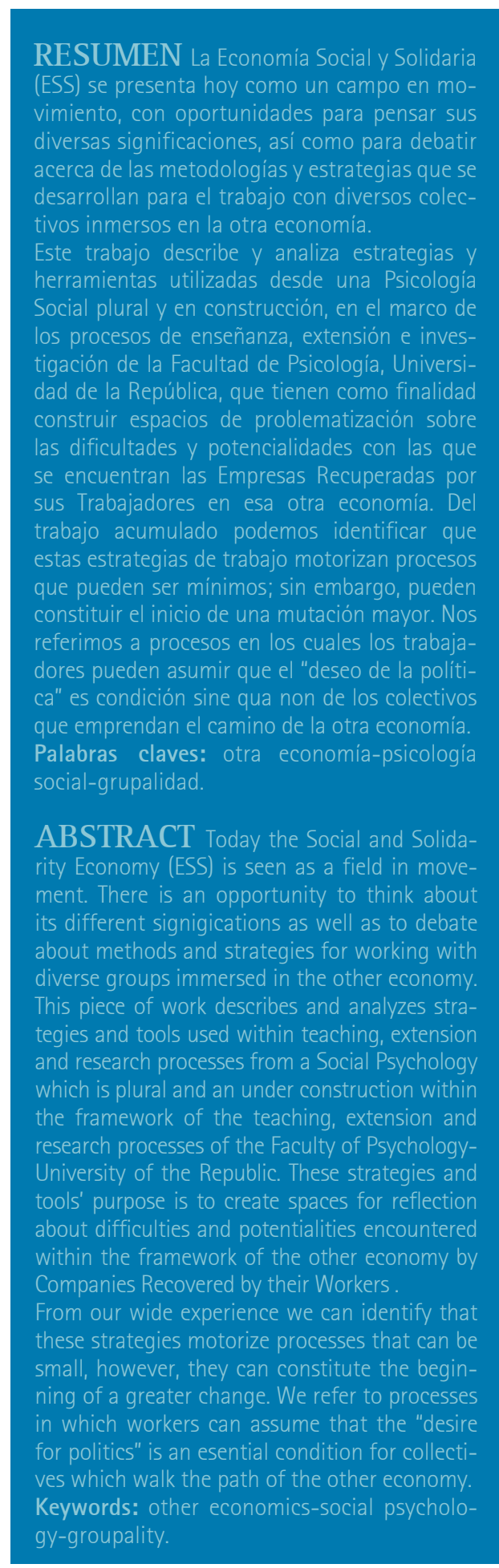

No podemos decir cómo será el mundo venidero, pero sabemos que será más justo. No lo sabemos porque lo sabemos, sino porque lo deseamos. El deseo potencia algo donde no hay nada. No se trata de un acto de fe, sino de una política. Política del deseo o deseo de la política: deseo que desea justicia e igualdad para el colectivo humano. Marcelo Percia, 2011: 231.

La Economía Social y Solidaria (ESS) se presenta hoy como un campo abierto y en construcción, capaz de alojar en su significación diversas expresiones del mundo asociativo, cooperativo y autogestivo. Históricamente diversas expresiones de la ESS han operado como mecanismo de inclusión social ante los procesos de exclusión provocados por diversas épocas de crisis del sistema capitalista. En los últimos tiempos han emergido nuevas prácticas colectivas que se interesan por construir nuevos modos sociales y relacionales de cara a producir "otra economía" (Coraggio, Laville, Cattani, 2013), dando surgimiento a nuevos actores que, sumados a los de mayor tradición, caracterizan a este sector particular de la economía (Guerra, 2012).

La emergencia de nuevas expresiones de la ESS abre el debate acerca de su significado y de las experiencias que pueden circunscribirse bajo esta denominación. Asimismo, da la oportunidad para elaborar, sistematizar y visibilizar estrategias de cara a fortalecer y multiplicar las experiencias existentes.

Las Empresas Recuperadas por sus Trabajadores (ERT) conforman una modalidad de la otra economía; son procesos sociales y económicos que nacen del proceso de quiebra o vaciamiento de una empresa de modelo capitalista, en el marco por el cual los trabajadores luchan por volver a ponerla en funcionamiento bajo formas autogestivas (Ruggieri, 2005). Los desafíos que los trabajadores de las ERT enfrentan se relacionan fundamentalmente con su matriz de surgimiento (Camilletti et al., 2005). El lugar simbólico del trabajo heredado (Weisz, 2012), signado por las lógicas del capital, dificulta la elaboración e invención de un presente sensible con la propuesta de la otra economía (Tommasino, 2017). 
De Prácticas y discursos

Universidad Nacional del Nordeste

Centro de Estudios Sociales

Año 7, Número 10, 2018, Octubre

ISSN 2250-6942

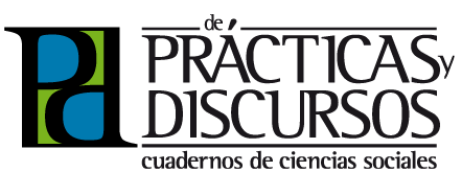

' Recibido el 12 de mayo de 2018 y aceptado el 20 de agosto de 2018.

${ }^{2}$ Facultad de Psicología, Universidad de la República. Correo electrónico: ntommasino@psico.edu.uy.
Este trabajo describe y analiza algunas estrategias y herramientas procedentes de la Psicología Social para la relación entre trabajadores, estudiantes y docentes universitarios con la ESS, en el marco de los procesos de enseñanza, extensión e investigación con una ERT. Moda Chic es una ERT cooperativa y autogestionada que produce y comercializa prendas de vestir femeninas en el centro de la ciudad de Montevideo. Los socios fundadores de este emprendimiento trabajaron más de 20 años en la empresa tradicional que por endeudamientos con el Banco República cierra en 2012. La patronal les deja la marca a tres trabajadores como forma de pago de todo el endeudamiento generado. En ese mismo año, once trabajadores deciden empezar a juntarse para reactivar la empresa. Como forma jurídica para funcionar, crean una cooperativa (de los once solo ocho finalizan este proceso) y de esta forma empiezan a construir un proyecto de recuperación de la unidad productiva y de autonomización del trabajo. En distintos momentos de este proceso, los trabajadores reconocen la difícil tarea que supone la gestión colectiva y algunas explicaciones que se formulan al respecto son: la escala del emprendimiento (ser pocos socios); la modalidad cooperativa que les impone la sistemática sensación de generar sus propios recursos a la velocidad que dicta el mercado capitalista y, al mismo tiempo, arriesgar por otras lógicas de producción y comercialización apostando al intercambio con otros emprendimientos cooperativos; la cantidad de años asumiendo un trabajo asalariado (que conlleva a la naturalización de esta forma de trabajo); la convicción de asumir un proyecto cooperativo relacionado a la inserción rápida en el mercado más que a promover otra forma del trabajo asociada a los valores de la "otra economía" y la poca trayectoria sindical del colectivo.

Algunas de estas dificultades fueron identificadas en procesos de trabajo conjunto con la Universidad de la República. Desde la Facultad de Piscología, particularmente, trabajamos con diversas herramientas provenientes de la Psicología Social que intentaron instalar un espacio grupal para problematizar las dificultades mencionadas -entre otras- y construir respuestas comunes a los problemas identificados. A grandes rasgos podemos decir que el uso de estas herramientas facilitó la problematización de la vida cotidiana con esta ERT. Del trabajo con las mismas podemos inferir que facilitan espacios de pensamiento para que los colectivos puedan preguntarse por su presente y asumir los 
desafíos de la gestión colectiva (Tommasino, 2017). Cuando los colectivos logran interrogar su presente de forma sistemática y generalizada, la pertenencia y la afiliación a los valores del trabajo asalariado entran en movimiento y se fortalece la pertinencia de un proyecto en el marco de la "otra economía".

La pregunta que guía este trabajo es cómo la Psicología Social desarrollada en el Río de la Plata actualmente contribuye a la creación de estrategias metodológicas para el trabajo con la ERT, en particular, y con la ESS, en general.

\section{UNA PSICOLOGÍA SOCIAL PLURAL Y EN CONSTRUCCIÓN}

Al preguntarnos qué es y qué hace la Psicologia Social (PS) se nos presenta una rápida solución, aunque tradicional y frágil. Solemos afirmar que se trata de una subdisciplina de la Psicología que se encarga de incursionar en lo social. Una solución un tanto más compleja es plantearnos que la PS está siendo, en movimiento y en construcción. "La Psicología Social Universitaria al ser desarrollada a través de las funciones universitarias se encuentra en permanente construcción, produciéndose y produciendo en el quehacer universitario y sus espacios colectivos" (Marqués, 2011: 40).

Esta particular mirada es sostenida desde la perspectiva Crítica y Construccionista de la PS (Ibáñez, 1992), así como desde la Psicología de la Vida Cotidiana y el Análisis Institucional. Estas perspectivas han posibilitado construir una tarea de la PS que se relaciona más que con resolver problemas con construirlos. El arte de construir problemas requiere abrir preguntas capaces de generar visibilidad sobre los diversos sentidos implicados en los acontecimientos (Etcheverry, 2004). Abrir visibilidad sobre los sentidos instala una forma particular de pensar, es una psicología que se pregunta más por "cómo" que por los "qué". Se pasa de la pregunta por la verdad a la pregunta por el sentido "en términos deleuzianos, se trata de atender al plano de la inmanencia, sustituyendo la monarquía causal por la causalidad inmanente; el sentido no precede sino que se constituye en el propio acto de su constitución" (Eira, 2005: 33). Pero, ¿cómo ha sido posible este pasaje?

Esta forma particular de la PS ha sido desarrollada fuertemente en el Río de la Plata, implicada en la creación de técnicas sobre intervenciones grupales vinculadas a los movimientos por la Salud Mental que se sostuvieron críticos ante el sistema de 
De Prácticas y discursos

Universidad Nacional del Nordeste

Centro de Estudios Sociales

Año 7, Número 10, 2018, Octubre

ISSN 2250-6942

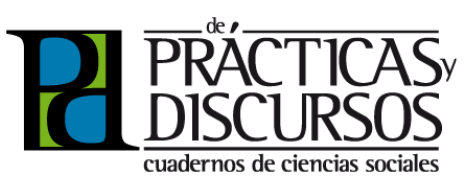

${ }^{3}$ Enrique Pichon Rivière fue psiquiatra y psicoanalista argentino, fundador de la Asociación Psicoanalista Argentina (APA) y cocreador de la Primera Escuela Privada de Psicología Social y del Instituto Argentino de Estudios Sociales (lades) en la década del 50.

${ }^{4}$ Roles dinámicos: portavoz, saboteador, líder, chivoemisario. Roles prescriptos: coordinador y observador.

${ }^{5}$ Afiliación, cooperación, pertenencia, pertinencia, comunicación, aprendizaje, telé. salud dominante. Los aportes de Enrique Pichon Rivière ${ }^{3}$ (19071977) son ineludibles en este sentido, para muchos fundador de la PS rioplatense, ya que posibilitó hacer el pasaje entre una concepción bio-psico-social a un concepción social e histórica de la subjetividad (Molas, 1999). La producción de Pichon Rivière genera un desvío en torno a los desarrollos grupalistas, nutrido de las perspectivas instituyentes y contrainstitucionales que emergían de "bullicios populares" en los años 60 y 70 . Interroga la vida cotidiana como escenario para establecer el carácter social e histórico de lo psíquico (Zuffiaurre, 2002). Pichon visibiliza la relación entre las formas de producir la existencia material, las experiencias, las prácticas que construyen subjetividad y el modo en que hombres y mujeres viven su vida.

La teoria del vínculo y la Técnica de Grupo Operativo (1948), creada en las circunstancias de una huelga en el Hospicio de las Mercedes en Buenos Aires, constituyen instrumentos para el trabajo en los espacios públicos (Fernández, 2008), concibiendo la situación grupal como medio y fin en sí mismo (Etcheverry, 2014). De su teoría destacamos el lugar central que le otorga a la tarea; esto significa que todo el proceso grupal girará en torno a una tarea manifiesta (objetivo del grupo) y otra latente (resolución de resistencias al cambio); por medio del análisis de los procesos de adjudicación y asunción de roles ${ }^{4}$ y de los vectores ${ }^{5}$ se elucida el proceso de producción grupal.

En la década de los 80 comienza a ubicarse otra dimensión para el abordaje teórico de la problemática grupal: las condiciones sociales e institucionales de las que los procesos colectivos emergen. El entramado social, la "exterioridad" grupal, es constitutivo de esa misma realidad grupal (Fernández, 1986). Comienza a circular un conjunto relevante de textos extranjeros, antes censurados, especialmente los que derivan del Análisis Institucional Francés de la mano de Lourau, Lapassade, Guattari, Oury y del Ilamado posestructuralismo, que tiene a Deleuze, Guattari, Foucault y Derrida como sus principales exponentes (AAW, 2012a). La entrada de estos textos se debe a una serie de fenómenos revolucionarios en términos políticos, epistemológicos, que dieron lugar al Mayo Francés, ofreciendo otras lecturas para el Río de la Plata (diferentes del lacanismo y del freudomarxismo). Permitieron mirar los fenómenos colectivos de otro modo, poniendo el foco en las redes simbólicas que sanciona la sociedad y definiendo una nueva estrategia: el Análisis Institucional. Las episte- 


\begin{abstract}
${ }^{6}$ Producto de estas corrientes de pensamiento cambian las formas de considerar algunas de las corrientes grupalistas específicas, el Psicodrama Moreniano, por ejemplo. Interesa particularmente en este trabajo porque utilizamos algunas de sus bases conceptuales y prácticas con los trabajadores de Moda Chic. Como dispositivo de la grupalidad se revoluciona su conceptualización y procedimientos cambiando sus alcances y desafíos. Véase Pavlovsky, Bouquet y Moccio (1991); y Pavlovsky, Kesselman y Frydlewsky (2007).
\end{abstract}

mologías posestructuralistas permiten hacer el pasaje de objeto discreto a la producción de redes transdisciplinarias y permiten crear nuevos pasajes de lo visible o lo enunciable en el campo de lo grupal ${ }^{6}$ (Fernández, 2008).

De este modo, los desarrollos de la PS del Río de la Plata se vieron enriquecidos y provocaron una tendencia "rupturista" del grupalismo (Percia, 2009), en contraposición a la tendencia hegemónica del psicoanálisis como dogma para pensar lo grupal y la Psicología en general. La tendencia de ruptura o desvío, como la denomina Percia (2009), rompe con el encierro producido en la institución psicoanalítica oficial. Se ubica como un desvío del modelo con un fuerte énfasis en el cambio social, posible, necesario e impostergable, teniendo los intelectuales y los profesionales un papel fundamental; "el trabajo intelectual, se decia, era una práctica para la construcción de otro lugar" (Percia, 2009: 24). Una particularidad de esta tendencia es la ocupación del espacio público para incidir en las transformaciones; el movimiento institucionalista, los equipos de salud y la diversificación de las prácticas son un ejemplo de ello. De esta forma, lo grupal comienza a configurar un campo de problemáticas per se (Fernández, 2008). Este cambio posibilita interrogarnos sobre lo grupal como un espacio estructurante de lo social-histórico; asimismo, la posibilidad de conceptualizar los grupos como nudos, espacios, procesos, dando lugar a los acontecimientos que alli se produzcan de forma singular, sin totalizar sus procesos y efectos. Podemos afirmar entonces que la PS rioplatense nace para reivindicar un cambio social comprometido con los procesos humanos y democráticos, atendiendo fundamentalmente a los procesos de subjetivación en cada época histórico-social, lo que conlleva a pensar no solo cómo formarse, enseñar, aprender, investigar, realizar extensión en " $x$ " condiciones, sino cómo llevar a cabo otra Psicología que colabore a resolver los problemas sociales de "x" período (Irrazábal, 2015).

Desde esta PS hemos intentando comprender los procesos colectivos que se producen en diferentes ámbitos de nuestro país, buscando el buen vivir de la población (Marqués, 2011). En los tiempos que corren cabe preguntarse por la vigencia del sentido humanizador y democrático de la Psicología Rioplatense en diálogo con la ESS hacia la construcción de un Uruguay social y económicamente más democrático e igualitario. En qué medida aporta la PS a la construcción de una ESS generalizada y fortalecida. 
De Prácticas y discursos

Universidad Nacional del Nordeste

Centro de Estudios Sociales

Año 7, Número 10, 2018, Octubre

ISSN 2250-6942

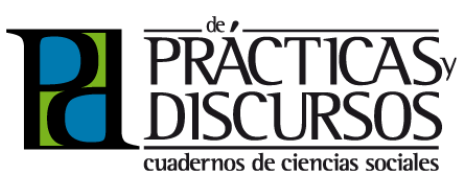

DIÁLOGOS ENTRE ECONOMÍA SOCIAL
Y SOLIDARIA Y PSICOLOGİA SOCIAL

En Uruguay, así como en la región, la crisis de principios del siglo XXI emerge como oportunidad (Tarrow, 1994) para el surgimiento de múltiples iniciativas vinculadas a la ESS: huertas comunitarias, merenderos, fábricas recuperadas y clubes de trueque. Si bien estas expresiones se han ido diluyendo con el tiempo, han transformado significativamente el sentido de la ESS, considerándola cada vez más como herramienta idónea para la inclusión social (Weisz, 2012; Rieiro, Weisz, Tommasino, 2018).

Existen muchas formas de denominar las experiencias vinculadas a la ESS en la región, economía solidaria, socio-economía solidaria, economía popular, nueva economía social, nuevo cooperativismo (Cruz, 2007); todas comparten ciertos principios básicos comunes que refieren a una equitativa distribución de los recursos priorizando el trabajo frente al capital; el sistema democrático participativo de organización del colectivo; el cuidado y la sustentabilidad medioambiental; la intercooperación y la intermediación sobre la base del comercio justo y el consumo responsable, entre otros.

Es posible hacer una genealogía del concepto y del surgimiento de la ESS en el Cono Sur a partir de los desarrollos teóricos de tres economistas latinoamericanos (Cruz, 2007). Estos desarrollos muestran parte de la disputa por su significación, así como acuerdos respecto a su potencial como herramienta, tanto para la inclusión como para la transformación social y cultural (sin dejar de evidenciar las dificultades para dicho proceso). Las diferentes perspectivas desarrolladas permiten observar distintas orientaciones, las que privilegian la ESS como alternativa, las que la plantean como una herramienta para gestionar lo social o bien aquellos que la plantean como proceso para la construcción de ciudadanía (Sarahu, 2009).

Del análisis de Cruz (2007) podemos decir que Razeto es quien por primera vez emplea en América Latina el término "economía solidaria" para denominar a un conjunto de experiencias de economía popular que en su práctica engendran la solidaridad. Afirma que estas experiencias no pueden asimilarse al cooperativismo, puesto que en él persiste un carácter subordinado al mercado capitalista, quedando desprovisto de la utopía social correspondiente a la estructuración del mercado y del Estado por medio de relaciones 


\begin{abstract}
${ }^{7}$ En Uruguay podemos vincular parte de la historia de la ESS con el desarrollo del cooperativismo, que se ha caracterizado por tener una continua relación con las organizaciones gremiales, la presencia y el papel del Estado fomentando u obstaculizando su desarrollo, la presencia del cooperativismo en el interior del pais y la defensa permanente de la autonomía y pluralidad como valores que lo sustentan (Bertullo et al., 2004).

${ }^{8}$ Fenómenos socioeconómicos que, por sus particulares lógicas de acción, sus racionalidades y los agentes movilizados, no pueden ser analizados por los estándares que dan cuenta de las lógicas privadas capitalistas, así como de la lógica público-estatal (Guerra, 2002 en Sarachu, 2009).
\end{abstract}

sociales innovadoras. Para el autor, tenemos el desafío de articular nuestras utopias en torno a un proyecto civilizatorio alternativo. Coraggio, por su parte, denomina "economía social" a un conjunto amplio de experiencias formales e informales, cooperativos, autogestionados, clubes de trueque, etc.; definida por el predominio del trabajo como componente de la reproducción. Propone una autonomización relativa de la economía popular con la economía capitalista y se pregunta cómo esta organización puede derivar en un modelo alternativo que priorice la vida y no el capital. Asimismo, plantea que estas experiencias locales se contraponen a lo global hegemónico, pero en tanto y en cuanto quieran derivar en una alternativa global deberán universalizarse. Finalmente, los aportes de Singer se caracterizan por pensar la "economía solidaria" primeramente como alternativa con relación al desempleo, más tarde la considerará un nuevo modo de producción en proceso de gestación. Incluye dentro de esta economía a los clubes de cambio, a las empresas autogestionarias y a las cooperativas populares. Las define como un conjunto de experiencias de acción innovadora en el campo de la inclusión social, como alternativas emancipadoras contraponiéndose a las políticas meramente compensatorias (Cruz, 2007).

Las primeras experiencias de ESS comienzan en Uruguay conjuntamente con el proceso de urbanización, apoyado fuertemente en la ola de los inmigrantes europeos que fomentan principios y valores del legado utópico socialista y anarquista (Errandonea y Supervielle, 1992), basados en los principios cooperativos internacionales e inspirados en la experiencia de "Rochdale" en Inglaterra ${ }^{7}$. La ESS ha tenido diversos escenarios socio-históricos para emerger, pero en cualquier caso su desarrollo ha estado ligado al impulso y retracción del Estado. Más allá de la diversidad de escenarios, motivos y formatos jurídicos que asumen las experiencias en clave de ESS, han demostrado ser una herramienta para la inserción socio-laboral conformando una práctica colectiva que opera positivamente frente al aislamiento y la vulnerabilidad, aunque persistan diversas dificultades para constituirse y consolidarse (Weisz, 2012).

Al día de hoy podemos decir que en Uruguay el campo de la ESS, también denominado socioeconomía de la solidaridad ${ }^{8}$ (Guerra, 2002), está conformado por la Economía Social (ES), la Economía Solidaria (EcoSol) y por los "autogestionarios" con elementos comunes y a la vez con marcadas diferencias. En la ES podemos 
De Prácticas y discursos

Universidad Nacional del Nordeste

Centro de Estudios Sociales

Año 7, Número 10, 2018, Octubre

ISSN 2250-6942

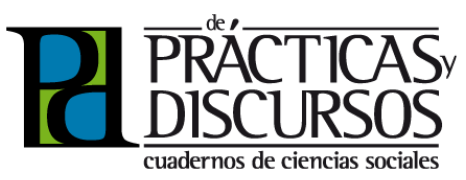

\begin{abstract}
${ }^{9}$ Nos referimos a pensadores de la Educación Popular (Paulo Freire, José Luis Rebellato), de la Teología de la Liberación (Leonardo Boff), de la Sociología Crítica (Orlando Fals Borda, Carlos Rodríguez Brandao, Pedro Demo) y de la Psicología de la Liberación (Ignacio Martín Baró) (AAVV, 2012b).
\end{abstract}

ubicar al cooperativismo, con debates en torno a cómo desarrollar modelos eficaces para su inserción en el mercado, los métodos de gestión y su capacidad para influenciar políticas públicas. En la EcoSol, el grado de institucionalización de las experiencias es menor que en el sector anterior y sus debates giran en torno al cambio social y la construcción de modelos alternativos. Finalmente, los que se identifican como "autogestionados" se conforman principalmente por las empresas recuperadas y se vinculan con el sindicalismo y la autogestión obrera (Rieiro, 2016). De esta clasificación podemos inferir que algunas de las disputas que enfrenta la ESS hoy refieren a las tensiones en el vínculo con el Estado, los desafios para la viabilidad, los alcances y los límites en tanto herramienta de inclusión social y estrategia hacia la transformación social (Weisz et al., 2018).

La experiencia indica que la matriz de surgimiento de cada experiencia, asi como la etapa en la que se encuentran, no determina necesariamente el alcance de las iniciativas económicas, sociales y culturales que se ubican en el campo de la ESS, sino que es en el devenir de la experiencia donde se entrelazan carencia y potencia (Weisz et al.,2018). Del mismo modo podemos decir que el potencial transformador de las diversas experiencias colectivas pasa por las conexiones que entre ellas establezcan; atender a sus vinculaciones y sus procesos de emergencia es central a la hora de pensar sus límites y posibilidades (Sarachu, 2009). ¿Pero, cuáles son las condiciones particulares de cada colectivo (deseantes, afectivas, relacionales) para que esto pueda ser posible?

La Psicología Social Comunitaria (PSC) en Uruguay ha estado próxima al desarrollo de las economías alternativas, populares, subalternas, etc. Esta proximidad se debe fundamentalmente al vínculo de la PSC con la extensión universitaria, a partir de lo cual se fortalece un posicionamiento político-académico donde el/la psicólogo/a comunitario/a prioriza la tarea de acompañar los procesos colectivos que tiendan a la generación de empoderamiento de grupos y comunidades en la defensa y ejercicio de sus derechos. Si bien esta psicología tiene tradiciones conceptuales y metodológicas distintas a la Psicología Social Grupalista ${ }^{9}$, hace parte de la caja de herramientas que utilizamos para el trabajo con los actores de la "otra economía". La PSC prioriza el trabajo con actores que conforman los movimientos sociales de América del Sur; los mismos pueden agruparse en cuatro grandes tejidos (se subdividen entre ellos, se separan o interconectan cuando toman par- 


\begin{abstract}
${ }^{10}$ Hemos transitado por la experiencia de construir y analizar dispositivos que generarán condiciones para mapear afectos en las experiencias de la otra economía. En la tarea de sistematizar la experiencia pudimos arribar a algunas potencias y debilidades de estos dispositivos que como orientación epistemológica se sustentaron en la Psicología Social que venimos describiendo.
\end{abstract}

tido en luchas especificas) (Jelin, 2003 en AAW, 2012b), a saber: 1. Tejido de los movimientos urbano-barriales, de obreros, de sindicalistas, de base, de las minorías sexuales; 2 . El tejido de los movimientos de mujeres -sindicalistas, feministas, de mujeres en el Estado, de mujeres en el Mercosur-; 3. El de los movimientos de derechos humanos; 4. El de los movimientos de gente y migraciones. En esta subdivisión podemos observar la presencia de actores de la "otra economía", así como orientaciones para el trabajo de la PSC, jerarquizando los problemas sociales en los cuales construye condiciones para intervenir.

En el Uruguay de 2002, esta psicología estuvo muy cerca de las diversas expresiones de la ESS que nacen como alternativas a la crisis socio-económica del país; el análisis de la PSC en esta coyuntura indicaba que estas expresiones se debatían entre constituirse en sujeto político o permanecer ligadas a las estrategias de sobrevivencia en el marco de las transformaciones en el mundo del trabajo (AAW, 2012b).

Como vemos, la ESS y estas vertientes de la Psicología Social comparten enunciaciones críticas sobre los modos en que pensamos e intervenimos lo social para su transformación. En este sentido, consideramos que uno de los mayores aportes como Psicólogo/as Sociales, vinculado al campo de la ESS, se centra en mapear y analizar experiencias que tengan una orientación distinta a las capitalocéntricas (Heras, 2011). Mapear y analizar circuitos de relaciones, afectos y dinámicas grupales que conforman el mundo de la otra economía constituye un análisis complementario a los diagnósticos (macro) sobre los alcances y limitaciones de la ESS para los procesos de transformación social ${ }^{10}$. Tenemos presente que los aportes provenientes de esta psicología inciden en la construcción de una mirada singularizante y cotidiana de los procesos colectivos que problematizan la mirada económica como representación hegemonizante y totalizante del análisis social.

HERRAMIENTAS PARA EL TRABAJO

CON LA ECONOMIAA SOCIAL Y SOLIDARIA

\section{a. Soporte político-metodológico}

Por lo antedicho podemos afirmar que la Psicología Social y la ESS promueven prácticas y herramientas alternativas para la or- 
De Prácticas y discursos

Universidad Nacional del Nordeste

Centro de Estudios Sociales

Año 7, Número 10, 2018, Octubre

ISSN 2250-6942

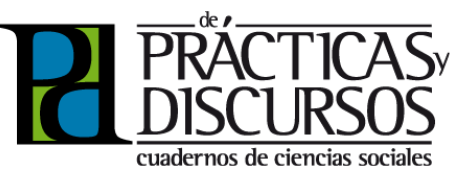

\footnotetext{
" Elucidar, para Castoriadis (2013), implica emprender un proceso de pensar lo que hacemos y saber lo que pensamos.
}

ganización de la vida. La crítica de la vida cotidiana, técnicas para abordaje de situaciones grupales, los estudios sobre las instituciones y sus componentes, la perspectiva de la subjetividad como una producción histórico-social y concepciones de la producción de saber y verdad, son algunas de las herramientas producidas por la Psicología Social. Se caracterizan por privilegiar los procesos y la noción de campo de problemáticas a estudiar (Marqués, 2011), y en el cruce con la ESS pensamos que nos ayudan a comprender "aspectos y elementos que se ponen en juego en toda creación y acción humana cuando el eje central es el trabajo en común de diversos actores" (Marqués et al., 2011: 2). Del trabajo con las mismas destacamos la posibilidad de indagar aspectos grupales, institucionales y organizacionales que permiten comprenderlas diversas situaciones que se suscitan cuando las personas se juntan. Para el estudio de la subjetividad con colectivos de la ESS particularmente, nos sostenemos en el método de Problematización Recursiva (Fernández, 2007), porque ofrece pistas para la indagación de sentidos estereotipados, prácticas (el lugar de lo no dicho) y la posibilidad de alojar lo inesperado (acontecimiento) con cada colectivo, a saber:

1. El trabajo para la identificación de insistencias de sentido implica acceder a cristalizaciones de sentido, rastrear operaciones que construyen los colectivos: repetición insistente de narrativas, institución de universos significantes morales, invisibilización de lo diverso, institución de regímenes de verdad, denegación de estrategias biopolíticas, operación recurrente de desplazamientos de sentido.

2. El trabajo para la indagación de prácticas implica el análisis de elementos extradiscursivos, el modo en cómo se organizan las prácticas, implica indagar respecto a los modos de actuar y de reflexionar.

3. El trabajo para la posibilidad de alojar lo inesperado implica hacer un análisis sobre las condiciones de los colectivos para alojar sus líneas de fuga (Deleuze y Guattari, 2006), así como producir condiciones para la emergencia de enunciaciones que de otra manera no tienen posibilidad de emerger.

El trabajo con estos componentes -y otros que pertenecen al método socioanalítico- (Lourau, 2007) nos ha permitido trabajar en la elucidación ${ }^{11}$ de los imaginarios sociales de colectivos que hacen parte de la otra economía. Esta forma de trabajo sostiene una particular concepción del campo de intervención. El mismo no es 
conformado por las personas, ni sus interacciones, ni el grupo, sino que lo conforma una grupalidad que se constituye como resultado de infinitas determinaciones sociales y políticas (Manero, 1990). Por ende, el cometido de nuestro trabajo no radica en analizar a los individuos, ni sus inconscientes, sino sus implicaciones grupales del "aqui y ahora" para relacionarlas con sus implicaciones sociohistóricas (González, 2002: 66). Desde esta concepción afirmamos entonces que el campo de intervención es un proceso elucidatorio colectivo sobre cómo actúan grupal y singularmente las determinaciones sociohistóricas.

Asimismo, este modo de trabajo nos ha permitido analizar los imaginarios grupales que un grupo o un colectivo instituye como propios. Si tomamos el caso de una ERT, por ejemplo, tendremos significaciones imaginarias que le son propias, generadas del propio proceso grupal.

Un grupo no solo es tributario de las producciones de significación más generales que la sociedad instituye: si fuera pertinente hablar de un imaginario grupal habrá que pensar en las figuras y formas que ese número numerable de personas inventa a lo largo de su historia común, para dar cuenta de sus razones de ser como colectivo: cobran aquí relevancia tanto sus mitos de origen como sus aspectos ilusionales de sus proyectos que, en tanto actualizaciones de deseo -se anuden o no se anuden al poder-, animan y motorizan sus prácticas. (Fernández, 1995: 2)

Cuando dinamizamos estos procesos en el marco de la ESS, se instala rápidamente un debate en el que es preciso detenerse: cómo recuperar el sentido político y afectivo de la técnica. En cuanto al sentido político, pensamos que es necesario revisar los modos dominantes de establecer la relación entre el saber técnico y la toma de decisión sobre sus alcances y consecuencias (siempre politicas); dado que muchas veces se niega la profunda relación y necesaria articulación entre lo técnico y lo político donde confluyen: técnicos comprometidos políticamente, políticas sustentadas técnicamente, entre otras implicaciones (Sarachu, 2013). En cuanto al sentido afectivo, consideramos que para producir procesos que promuevan la creación de un pensamiento nuevo es necesario que el técnico establezca relaciones de confianza, devenir un recurso presente generador de una práctica afectiva minoritaria (Deleuze, 1992), un intercesor; 
De Prácticas y discursos

Universidad Nacional del Nordeste

Centro de Estudios Sociales

Año 7, Número 10, 2018, Octubre

ISSN 2250-6942

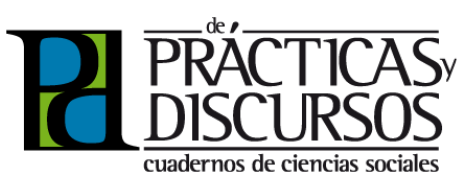

não como um especialista (consultor, assessor, psicólogo ou mesmo restrito à função de pesquisador), mas como alguém que desliza no movimento desse coletivo sem ser o agente e nem a referência desse movimento, embora possa, com sua ação como um de seus integrantes, suscitar a reflexão coletiva que desencadeie o movimento. (Andrade, 2013: 8)

Cuando elucidamos el sentido político y afectivo de la(s) técnica(s), visualizamos que estos motorizan gran parte de nuestro trabajo con los colectivos de la ESS. Asumir como propia la necesidad de los colectivos, identificarnos en sus proyectos -que no significa asumirnos en lo idéntico- es una forma de involucrarnos desde los afectos y necesidades comunes para el trabajo compartido.

\section{b. Del con-texto}

A vuelo de pájaro, nos interesa situar la emergencia de las ERT en la región y en Uruguay para entender el enclave de sus dificultades y el pedido de intervención que realiza Moda Chic a la Facultad de Psicología.

Las ERT como una expresión o modalidad de la "otra economía" han surgido como consecuencia de la desestabilización política, de la precarización del trabajo principalmente por la apertura económica. Nacen de empresas deficitarias, públicas y privadas (Martí, 2010). En su mayoría han adoptado el modo cooperativo para llevar adelante la autogestión, y como cooperativas es importante evaluar su matriz de surgimiento y su modalidad (productiva).

En Uruguay, las ERT han encontrado diversos escenarios sociohistóricos para emerger, resistir y reinventarse. Podemos situar cuatro etapas históricas de emergencia que condicen con impulsos y frenos de la ESS en nuestro pais:

1. La crisis del modelo de industrialización de importaciones (1950-1970).

2. El proceso de liberación de la economía nacional que como resultado tuvo la desindustrialización (1980-1990).

3. La quiebra del modelo neoliberal (fines de 1990) (Martí et al., 2013).

4. La llegada de los gobiernos progresistas (Rieiro, 2016), momento en el cual rebrota el cooperativismo, la autogestión en particular, y comienzan a manifestarse otras expresiones de la ESS.

En el escenario actual, las ERT tienen como desafío reinventarse 
en sus modos organizativos para autonomizarse de los apoyos estatales (Hudson, 2011; Novaes y Sardá de Farias, 2009) que si bien son débiles y fragmentarios (Martí, 2010), son apoyos clave para el sustento económico. Esta situación configura una contradicción para los colectivos de la otra economía, porque la ayuda estatal, al mismo tiempo que colabora con muchas experiencias, induce procesos cooperativos desafiliados y acríticos con los principios y valores de la ESS. Este efecto puede observarse cuando la ayuda del Estado encorseta a los colectivos de la ESS en modos de producción y comercialización con lógicas capitalistas, cooptando otros modos de organización e intercambio. El apoyo estatal incentiva a que los emprendimientos disputen el mercado, desmovilizando el sentido de la lucha y generando un "sentido estatal de la autogestión" (León, s/f). Dentro de la ESS hay posiciones que afirman la posibilidad del diálogo entre la ESS y el Estado, otras afirman su imposibilidad, dado que el Estado siempre la restringirá a lo empresarial, es decir en una microautogestión. En este sentido es importante permanecer críticos ante los colectivos que quedan librados a una red que opera sobrecodificando líneas de segmentariedad (duras, de representación: Estado, capital) sin poder construir otras que tienden a generar flujos mutantes que escapan al código (Deleuze y Guattari, 2006) (relaciones solidarias y de intercooperación). Las ERT, que también se denominan autogestionadas, ocupadas y tomadas, usurpadas, reconvertidas, de gestión obrera (Martí et al., 2013), dentro de la ESS tienen obstáculos y desafíos especificos. La experiencia acumulada indica que el principal desafio está en poder reformular sus agenciamientos de deseos y crear nuevos modos de articular sus deseos y sus historias, es decir, inventar sus políticas (Fernández et al., 2004) en el marco de otras lógicas no capitalistas. Dado el legado de la empresa madre capitalista, la significación del lugar simbólico del trabajo (Weisz, 2012) tensiona la propuesta que hace la ESS sobre el desarrollo integral de lo humano. Como consecuencia, estos colectivos necesitan objetivar los modos mediante las cuales se han subjetivado, pero ello requiere de condiciones donde puedan efectuar dicho análisis (Mendy, 2010).

La experiencia indica que la red de la ESS constituye un nuevo entramado para resignificar la subjetividad construida por el trabajo asalariado; producen "un afuera" humanizado y solidario, rompiendo con la lógica mercantil aplicada al intercambio entre 
De Prácticas y discursos

Universidad Nacional del Nordeste

Centro de Estudios Sociales

Año 7, Número 10, 2018, Octubre

ISSN 2250-6942

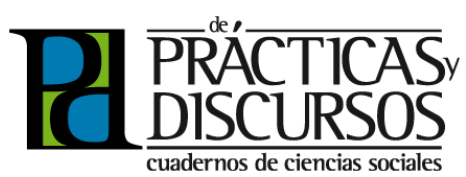

las cosas, pero sobre todo a las relaciones entre las personas. Pliegan modos de ser y estar distintos a los modos del capital (Tommasino, 2017).

Tras el avance de las políticas neoliberales en la región, el escenario se vuelve más incierto y algunos colectivos de la ESS quedan aún más a la intemperie. Esta coyuntura nos exige como técnicos estar más críticos y atentos a los efectos de "la razón indolente de la que somos hereditarios única, exclusiva y suficiente que bloquea las opciones que se construyen entre la relación social establecida desde la universidad pública -a la cual pertenecemos- con los sujetos de la otra economía" (Rieiro, Weisz, Tommasino, 2018: 8). ¿Cómo cuidar los logros y potencialidades de estas experiencias? ¿Cómo desarrollar acompañamientos críticos de los criterios mercantilizados y productivistas exigidos por los globalizados estándares universitarios? ¿Cómo sostenerse atentos a no trasplantar la ciencia del capital en nuestros acompañamientos?

\section{c. De los encuentros}

Puesto que es necesario ubicar las herramientas en torno a procesos de trabajo para singularizar sus efectos, tomamos la experiencia con Moda Chic, una ERT cooperativa y autogestionada de Montevideo. En 2014, en el marco del acompañamiento que la Unidad de Estudios Cooperativos del SCEAM realizaba con los trabajadores, se logran identificar dificultades en el pasaje de trabajadores asalariados a independientes. Se decide solicitar apoyo a la Facultad de Psicología para acompañar dificultades específicas de la práctica cooperativa y se construye un espacio grupal para enfrentar los desafíos de asumir la gestión colectiva. El proceso que se puso en marcha con los trabajadores tuvo como objetivos:

1. Reflexionar sobre la práctica.

2. Reconocer y potenciar los saberes y prácticas autónomas de los trabajadores.

3. Documentar relatos de los trabajadores, sus memorias, afectos y las implicaciones sociales, políticas, económicas como organización.

Los encuentros de trabajo fueron grupales con una frecuencia quincenal de dos horas de duración. La situación grupal fue el soporte para compartir recorridos singulares, para rescatar la memoria y el futuro de ese colectivo. Adviene dispositivo porque 
se construye como un espacio de interrogación que tiene como finalidad alterar lo heterónomo y rescatar aquello que se resiste a instituirse para convertirlo en fuerza colectiva de transformación. Lo grupal, en este sentido, se vuelve un espacio fértil para disputar lo común como sostén para las decisiones colectivas (Bonano, 2008).

La Técnica Operativa de Grupo y las Técnicas de Acción Sociométricas y Sociodramáticas generaron una superficie para encontrarse y compartir -múltiples- relatos sobre la práctica a partir de los cuales emerge la visualización de la diferencia como cualidad humana. Este aspecto es crucial para los objetivos planteados, porque desarticula las "lógicas de lo uno" -lógica de los relatos dominantes, totalizantes y homogenizantes caracteristicas del trabajo asalariado- e instala la "lógica de la multiplicidad" (Fernández, 2007) (lógica de los relatos minoritarios, rizomática y multiplicadora, característica de la otra economía). Pudimos observar que en Moda Chic conviven ambas lógicas, permanecen en tensión, articulándose y desarticulándose. Uno de los desafios del espacio grupal fue construir enunciación sobre cómo operan en el cotidiano vivir de los trabajadores, cómo condiciona el presente del colectivo y en qué medida permiten construir un futuro sensible con las lógicas de la ESS.

Con la Técnica Operativa de Grupo fue posible trabajar distinguiendo enunciados sobre sus dificultades, potencialidades, implicaciones afectivas, políticas, institucionales. La tarea de la coordinación, en este sentido, tuvo el desafío de violentar el sentido común de la enunciación para trabajar en un proceso problematizador y transformador. De esta manera, pudimos comprender las distintas dimensiones de la grupalidad que están presentes en el colectivo de trabajadores, estas son:

1. Dimensión grupal: esta dimensión refiere a los procesos dinámicos que se producen en cualquier situación grupal. Las personas en esta situación se ven ligadas por una relación social que construye un soporte para el desarrollo de la propia situación grupal. Los análisis de dinámicas grupales han variado en las distintas teorizaciones sobre lo grupal; para el trabajo con la ERT tomamos fundamentalmente aquellos creados por Pichon Rivière (1982), para el cual todo el proceso grupal gira en torno a una tarea manifiesta y otra latente que, sumado al análisis de los procesos de adjudicación y asunción de roles y 
De Prácticas y discursos

Universidad Nacional del Nordeste

Centro de Estudios Sociales

Año 7, Número 10, 2018, Octubre

ISSN 2250-6942

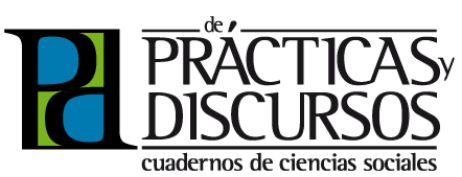

de los vectores, elucida el proceso grupal. Describimos a continuación parte del análisis de la dinámica grupal realizada con los trabajadores de Moda Chic, poniendo énfasis en el análisis vectorial, puesto que visibiliza la evolución de la tarea.

Comunicación, el análisis de este vector hizo evidente que la comunicación era una de las dificultades asumidas por el colectivo, entendiendo que varios de los malestares se fundan en la modalidad comunicacional instituida. Unos de los efectos del espacio grupal, constatado en los relatos de los trabajadores, refiere a la posibilidad de construir otros modos comunicacionales, poder hablar y ser escuchado, que entre otras cosas permite desarticular los "corrillos" como modalidad preferida por los trabajadores y enfrentarlos con la necesidad de construir otras formas comunicacionales acordes a los valores de la ESS.

La afiliación, como vector, muestra que la herencia de la fábrica debilita la posibilidad de afiliarse a un proyecto cooperativo; sin embargo, las relaciones de intercooperación y los cursos que ellos mismos brindan a la comunidad fortalecen el vector. Del espacio de trabajo emerge que en la medida que problematizan los efectos de su matriz y desarrollan otro sentido colectivo del trabajo se fortalece la afiliación a la cooperación. Del mismo modo, la pertenencia de los integrantes y la pertinencia del proyecto se fortalecen según las tareas que puedan construir como grupo y las oportunidades que se dan para que algunos roles circulen.

Si bien en una cooperativa esperamos que el vector de cooperación esté presente como una característica propia del accionar en sus distintos ámbitos (Marqués, 2009), en este caso existen momentos de mayor y menor cooperación entre los trabajadores y con la comunidad a la que pertenecen. Este análisis hace que se desvanezca la idea de que la cooperación es una acción plena, constante, generalizada y trascendente. Por el contrario, emerge como una práctica que es necesaria construir sistemática y cotidianamente, con contradicciones, y que es inmanente al desarrollo de la propia práctica cooperativa.

El vector de aprendizaje muestra que como colectivo deben poner a jugar todo lo aprendido durante la experiencia del oficio, pero a la vez deben disponerse a aprender otros saberes que son necesarios para la gestión colectiva. Del espacio de trabajo emerge que la circulación de información es una dificultad; el proceso de asunción y adjudicación de roles evidencia la predisposición 
a preservar personas y lugares reproduciendo una forma de la tarea (asalariada) y dificultando el aprendizaje colectivo. En ese sentido, la tarea de "aprender a pensar" se ve impedida porque depositan las ansiedades (grupales) en un chivo emisario y las dificultades en un liderazgo; de esta menara preservan al grupo de asumir sus problemas colectivos. Visualizamos que el espacio grupal instala condiciones en este sentido, generando una superficie nueva para que los trabajadores se encuentren desde otro lugar y puedan experimentar sus diferencias; condición sine qua non para el aprendizaje.

Por último, el análisis de la telé (clima afectivo) muestra que la telé negativa deriva de las condiciones de emergencia, la experiencia de estar "obligados" a convivir para recuperar su fuente de trabajo. Pudimos distinguir que parte de esta telé negativa deriva de las implicaciones institucionales no elaboradas como colectivo. Este fue uno de los vectores transversales más trabajados, puesto que en él depositaban casi la totalidad de las dificultades colectivas.

2. Dimensión institucional: para el análisis de esta dimensión tomamos aportes del Análisis Institucional; del Psicoanálisis tomamos el concepto de "implicación" porque nos permite pensar el "conjunto de relaciones, conscientes o no, que existen entre el actor y el sistema institucional" (Lourau, 2007). De esta manera es posible problematizar con los colectivos el coeficiente de transversalidad (Guattari, 1976) de los actores involucrados en sus prácticas, ampliarlo y descentrar la mirada de los modos habituales y hegemónicos. La institución pensada como "árboles de decisiones lógicas, que regulan las actividades humanas, indicando lo prohibido, lo permitido y lo indiferente..." (Baremblitt, 2005: 156) constituye una dimensión fundamental para comprender los procesos grupales. Del análisis de esta dimensión podemos comprender los procesos de producción en su condición productiva, reproductiva o antiproductiva. Entendiendo por producción productiva todo aquello que pueda ser generador de lo nuevo, lo que la "utopía activa persigue", aquello capaz de procesar las innovaciones insólitas, es el devenir, es la metamorfosis. Se trata de que la producción productiva de todo grupo no quede capturada por formas reproductivas (estereotipadas) y antiproductivas (de captura) de la producción (Baremblitt, 2005). 
De Prácticas y discursos

Universidad Nacional del Nordeste

Centro de Estudios Sociales

Año 7, Número 10, 2018, Octubre

ISSN 2250-6942

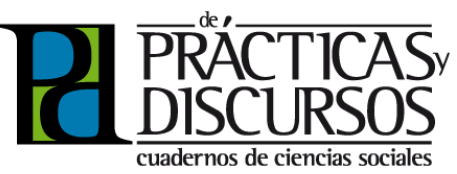

Del trabajo realizado con Moda Chic podemos decir que esta dimensión arroja visibilidad sobre los sentidos y prácticas instituidas por la fábrica, creadas bajo lógicas disciplinarias y mercantiles, que derraman hacia la producción deseante del colectivo. Esto implica que el colectivo queda muchas veces en una práctica reproductiva, que refiere a copia/imitación de antiguos saberes y prácticas de la fábrica impidiendo los devenires, acontecimientos, lo nuevo. Esta práctica deja en evidencia que la matriz de surgimiento del colectivo se instala recursivamente en su vida cotidiana y es necesario interrogarla. Interrogarla hace posible problematizar el lugar determinante de lo social-histórico y la posibilidad de transformarlo. Esta dimensión arroja visibilidad sobre los procesos de producción de subjetividad y subjetivación, de los que son parte los trabajadores, permitiendo la invención de líneas de significación para lo que acontece (Etcheverry, 2014). Podemos decir entonces que dimensión rastrea y problematiza sentidos cristalizados, inmóviles, significantes grupales y sociales, narrativas insistentes que sostiene el colectivo.

3. Dimensión organizacional: esta dimensión se caracteriza por las formas concretas y materiales que nos damos los humanos para responder a las necesidades sociales que identificamos (Marqués et al., 2005). Si tomamos el caso de las cooperativas, por ejemplo, decimos que son organizaciones constituidas por personas, reglamentos, recursos materiales, el establecimiento, entre otras, que hacen posible su trabajo cotidiano y materializan la institucionalidad a la que pertenecen. Especificamente, el análisis de esta dimensión con Moda Chic permitió visualizar la materialización de algunos instituidos, así como la posibilidad tangible para movilizarlos. Identificar la dimensión material concreta de la práctica expresada en la organización del tiempo y del espacio, formas y lugares que se dan los trabajadores para su labor, fue fundamental para trabajar en clave de resignificación de experiencias.

Por otro lado, con la Sociometría y el Sociodrama como técnicas fue posible visualizar que la acción deviene un buen soporte para trabajar la resignificación grupal. Los mitos colectivos, prácticas, formas de ser y de estar con las que tomaban contacto, entraban en un circuito de enunciación-movilización. Es decir, los elementos que eran enunciados como problema-conflicto al dramatizarlos visualizábamos otras aristas del problema, los afectos que 
De Prácticas y discursos

Universidad Nacional del Nordeste

Centro de Estudios Sociales

Año 7, Número 10, 2018, Octubre

ISSN 2250-6942
ECONOMÍA SOCIAL Y SOLIDARIA EN MOVIMIENTO: DIÁLOGOS

Y APORTES DESDE UNA PSICOLOGÍA SOCIAL PLURAL

SOCIAL AND SOLIDARITY ECONOMY IN MOTION: DIALOGUES

AND CONTRIBUTIONS FROM A PLURAL SOCIAL PSYCHOLOGY

\begin{abstract}
12 "Expresan la movilidad propia del tiempo, traen consigo mutaciones y singularizaciones; todo cambia en el acontecimiento (Teles, 2006).
\end{abstract}

\footnotetext{
${ }^{13}$ Moreno (1993: 153) afirma que para experimentar un momento como "sui generis" es preciso que se generen determinadas circunstancias: 1. Debe tener lugar un cambio en la situación; 2. el cambio debe ser suficiente para que el sujeto perciba la experiencia de novedad; 3 . esta percepción implica actividad de parte de la persona, un acto de estimulación para un estado espontáneo.

${ }^{14}$ El trabajo con esculturas permite poner en acto sensaciones, emociones, imágenes e historias que nos hablan de relaciones, significaciones sociales imaginarias y de instituidos grupales y sociales. Se vuelve un analizador para identificar el "drama del momento" (drama del momento social, institucional, grupal) de un colectivo en particular.
}

generaba y las salidas posibles a construir. Las técnicas dramáticas tienen el objetivo de poner en evidencia sistemas represivos y conductas que estos fomentan; detectar y enfrentar situaciones de injusticia social y de diferencias sociales; investigar conductas autoritarias dentro y fuera de las instituciones; analizar los roles sociales, los emisores de las normas que en defensa de sus propios intereses imponen roles no relacionados con el interés de la comunidad (Pavlovsky, Bouquet, Moccio, 1991: 8).

Con este trabajo logramos construir una superficie de cuerpos afectados para identificar los sentidos que emergen como necesarios a resignificar. Estas herramientas eran utilizadas principalmente cuando requeríamos una reorientación del trabajo grupal, de modo que los trabajadores tuvieran otras experiencias para alojar lo inesperado, la emergencia de un acontecimiento ${ }^{12}$, es decir, experimentar un momento como "sui generis"13. Los trabajadores rescatan de estas herramientas:

- El efecto alegre y distendido de la acción.

- La acción como otra posibilidad para generar pensamiento, pensamiento novedoso sobre un hecho.

- Construir una imagen distinta de sí.

- Decir sin hablar.

- La posibilidad de interrogarse sobre lo que les pasa y sobre su presente.

- La posibilidad de identificar tres momentos centrales del proceso y las sensaciones diversas que emergen al dramatizarlo con esculturas ${ }^{14}$

\section{ABRIR SENTIDOS Y PRODUCIR DESEO}

Como vemos, estas herramientas dispusieron un circuito de enunciación-escucha-pensamiento y cambio.

El proceso de trabajo tuvo como tarea elucidar las diferentes demandas que subyacen al pedido formulado por los trabajadores de Moda Chic. El pedido implicaba trabajar el malestar producto del mal relacionamiento entre ellos. Formulado así, pensamos que uno de los mecanismos que operaba en este colectivo es la psicologización de los problemas. Este mecanismo se construye a partir de la desconexión entre las personas y los procesos sociales e históricos. Los análisis que insisten en despsicologizar los problemas permiten identificar las afectaciones institucionales 
De Prácticas y discursos

Universidad Nacional del Nordeste

Centro de Estudios Sociales

Año 7, Número 10, 2018, Octubre

ISSN 2250-6942

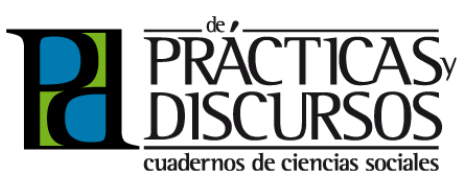

que han diagramado y construido los sentidos versus las tradiciones que privilegian lo interior-individual y lo "familiarista" (Fernández, 2008) para explicar y evaluar las dificultades (laborales) de los colectivos. Denegar las afectaciones institucionales edipiza sus rebeliones y sumisiones, exiliando su política (Fernández y Herrera, 1991) e impidiéndoles hacer un análisis crítico sobre su entramado relacional social e histórico. A partir de este análisis fue posible visualizar que el relacionamiento entre ellos se inscribe en el universo de dificultades que enfrentan las ERT en general y en las condiciones que, como colectivo, en particular, se dieron para tramitarlas. Por lo tanto, fue posible despsicologizar el problema.

Conforme pudimos visualizar la fuerza social histórica de la empresa capitalista de procedencia, y entender cómo esta historia permea e incide en el presente del colectivo, se instalaron mayores condiciones para encontrarse y hablar. La posibilidad de construir enunciación (un hablante) alli donde parecia imposible escucharse fue lo que posibilitó interrogar su presente y proyectar un futuro común. El lenguaje alojó la posibilidad de tomar contacto con las diversas significaciones que sostienen al colectivo y cuando esto no fue suficiente, nos sostuvimos en las técnicas de acción que generan otras condiciones para mostrar e interrogar los cambios que desean enfrentar.

Pensamos que uno de los efectos más importantes del trabajo fue la capacidad de poner en movimiento el pensamiento, ya que, como dice Deleuze (2015), solo pensamos si "algo nos da qué pensar" o si tenemos las condiciones para ello, debiamos hacerle espacio y tiempo. Pensar implicaba, a su vez, ponerse en contacto con sus propios recursos (De La Aldea, 2015) y con los cambios que son importantes producir como colectivo. El cambio, como problemática grupal, implica que todo grupo se adecúe activamente a una realidad cambiante, es decir, a su capacidad de redefinir la tarea colectiva según las situaciones que enfrentan; definir los cómo y con quienes, es de algún modo la redefinición política de lo común que como "puntada provisoria en grupos colectivos y comunidades" permite la acción conjunta (Percia, 2017: 285).

Entonces, esta es la Psicología Social que queremos, que sea capaz de acompañar colectivos que requieren definir sus problemas e inventar sus políticas, hacer autoanálisis (Baremblitt, 2005). Y porque este proceso nunca es en solitario (es colectivo, 
De Prácticas y discursos

Universidad Nacional del Nordeste

Centro de Estudios Sociales

Año 7, Número 10, 2018, Octubre

ISSN 2250-6942
ECONOMÍA SOCIAL Y SOLIDARIA EN MOVIMIENTO: DIÁLOGOS

Y APORTES DESDE UNA PSICOLOGÍA SOCIAL PLURAL

SOCIAL AND SOLIDARITY ECONOMY IN MOTION: DIALOGUES

AND CONTRIBUTIONS FROM A PLURAL SOCIAL PSYCHOLOGY
${ }^{15}$ El desafío de compartir el trabajo, los medios de producción, de superar la apropiación privada de la producción social de la riqueza, de la separación entre los que hacen y deciden (Sarachu, 2012). en multiplicidad de relaciones -sociales y solidarias-), necesitamos instalar las condiciones para reencontrarnos colectivamente. Hacer autoanálisis incita a recuperar el "deseo de la política" (Percia, 2011), una tarea que no es para nada sencilla y que precisa engendrar -otros- procesos minúsculos pero complejos. Este trabajo quiere contribuir en esta línea, identificar una serie de procesos que repolitizan los encuentros y los deseos de los colectivos; son de algún modo potencias de los dispositivos con lo que trabajamos.

1. Problematización de la vida cotidiana: develar cómo algo ha conseguido instalarse como aproblemático. Problematizar la vida cotidiana de la ESS es acompañar en las formulaciones de los problemas humanos y colectivos.

2. Producción de la diferencia: reconocer la diferencia como cualidad de los colectivos humanos, construir condiciones para habitarla y producirla.

3. Emergencia de la rebeldia: hacer emerger la rebeldía, movimiento del descontento con lo conocido, necesario para producir un pensamiento nuevo.

4. Creación de nuevas significaciones: sostener la emergencia de nuevas producciones a partir de un proceso creativo y espontáneo que habilite elaborar críticamente el pasado para producir imaginación política, utopía compartida.

Pensamos que estos procesos, para algunos colectivos de la ESS, tienen el potencial de motorizar la resignificación del lugar simbólico del trabajo, así como problematizar las diversas dificultades que enfrentan para afiliarse a los principios de la otra economía. Asimismo, estos procesos aportan a la generación espacios de formación y pensamiento para que los colectivos puedan asumir los desafíos y rupturas ${ }^{15}$ (Sarachu, 2012) que exige todo trabajo asociado.

Nos queda como gran desafío mantener vivas las herramientas que utilizamos, esto significa revisarlas, actualizarlas, modificarlas, para que contribuyan críticamente a los procesos de una Economia Social y Solidaria que está siendo.

BIBLIOGRAFÍA

AAVV (2012A). Programa Estudio de las Formaciones Subjeti- 
vas. Instituto de Psicología Social. Montevideo: Documento de circulación interna.

AAVV (2012B). Programa de Psicología Social Comunitaria. Instituto de Psicología Social Montevideo: Documento de circulación interna.

ANDRADE, M. (2013). 0 encontro da loucura como o trabalho na economia solidaria: a produção de práxis de pré-incubagem através do dispositivo intercessor na Saúde Mental.Tesis de Doutorado. Universidade Estadual Paulista, Faculdade de Ciências Letras de Assis, São Paulo, Brasil.

BAREMBLITT, G. (2005). Compendio de análisis institucional y otras corrientes. Buenos Aires: Madres de Plaza de Mayo.

BERTULLO, J.; ISOLA, G.; CASTRO, D. Y SILVEIRA, M. (2004). El cooperativismo en Uruguay. Cuadernos de Rectorado, vol. 22. Montevideo: CSEAM, Universidad de la República.

BONANO, 0. (2008). Grupo y dimensión colectiva de las prácticas. En Bozzolo, R. y Bonano, 0. (comps.) El oficio de intervenir. Políticas de subjetivación en grupos e instituciones (pp. 145155). Buenos Aires: Sin Fronteras.

CAMILLETTI, J.; RODRÍGUEZ, M. Y MARTí, J.P. (2005). Cooperativas de trabajo en el Cono Sur. Matrices de surgimiento y modelos de gestión. Unircoop,3(1), 32-56.

CASTORIADIS, C. (2013). La institución imaginaria de la sociedad. Buenos Aires: Tusquets.

CORAGGIO, J.L.; LAVILLE, J.L. Y CATTANI, A.D. (COORDS.) (2013). Diccionario de la otra economía. Universidad Nacional General de Sarmiento. Buenos Aires: Los Polvorines.

CRUZ, A. (2006). La construcción del concepto de economía solidaria en el Cono Sur. Revista de Estudios Cooperativos, 16(1), 7-27.

DE LA ALDEA, E. (COMP.) (2015). Los talleres. Cuidar al que Cuida. Cuaderno N³, Año 3. Buenos Aires: Los talleres. 
DELEUZE, G. (2015). Curso sobre Foucault, Vol. 3. La subjetivación. Buenos Aires: Cactus.

DELEUZE, G. (1992). Os intercessores. En Deleuze, G. (ed.) Conversações (pp. 151-168). Rio de Janeiro: Ed. 34.

DELEUZE, G. Y GUATTARI, F. (2006). Mil Mesetas. Capitalismo y esquizofrenia. Valencia: Pre-textos.

Eira, G. (2005). La verdad, la certeza y otras mentiras. Montevideo: Psicolibros.

ERRANDONEA, A. Y SUPERVIELLE, M. (1992) Las cooperativas en el Uruguay. Análisis sociológico del primer relevamiento nacional de entidades cooperativas. Montevideo: Fundación de Cultura.

ETCHEVERRY, G. (2004). Vida cotidiana y salud enfermedad: modalidades de la producción subjetiva. En Folle, M. y Protesoni, A. (comps.) (2005). Tránsitos de una Psicología Social. Montevideo: Psicolibros.

ETCHEVERRY, G. (2014). Relación asistencial y grupalidades en la enfermería hospitalaria: el caso del Centro Hospitalario Pereira Rossell. Tesis de Maestría. Universidad de la República, Facultad de Psicología.

FERNÁNDEZ, A.M. (1995). La invención de significaciones y el campo grupal. Revista Subjetividad y Cultura, 5. México.

Fernández, A.M. (2007). Las Lógicas Colectivas. Imaginarios, cuerpos y multiplicidades. Buenos Aires: Biblos.

(2008). Notas para una genealogía. Buenos Aires: Eudeba.

FERNÁNDEZ, A.M. Y HERRERA, L. (1991). Laberintos institucionales. En Espacio Institucional (pp. 67-86). Buenos Aires: Lugar Editorial.

FERNÁNDEZ, A.M.; LÓPEZ, M.; OJÁM, E. Y IMAZ, X. (2004). Los imaginarios sociales. Del concepto a la investigación de campo. Tramas (22), 145-179.

GONZÁLEZ, F. (2002). Análisis institucional y socioanálisis. 
Tramas, (18/19), 51-72. Recuperado de: http://132.248.9.34/hevila/TramasMexicoDF/2002/no18-19/2.pdf

GUATTARI, F. (1976). Psicoanálisis y transversalidad. Crítica psicoanalítica de las instituciones. Buenos Aires: Siglo XXI.

Guerra, P. (2002). Socioeconomía de la solidaridad. Montevideo: Editorial Nordan-Comunidad.

(2012). La legislación sobre economía social y solidaria. Casos latinoamericanos y europeos. Documento de Trabajo $N^{\circ}$ 4. Montevideo: Facultad de Derecho. Universidad de la República.

HERAS, I. (2011). Pensar la autonomía. Dispositivos y mecanismos en proyectos de autogestión. Intersecciones en comunicación. Recuperado de: http://www.ridaa.unicen.edu.ar/xmlui/ handle/123456789/719

HUDSON, J.P. (2011). Acá no, acá no me manda nadie: empresas recuperadas por obreros. Buenos Aires: Tinta Limón.

IBÁÑEZ, T. (1992). Como se puede no ser construccionista hoy en dia. Revista de Psicoterapia, (12), 245-257.

IRAZÁBAL, E. (2015). Proyecto Profesor Titular Grado 5 para el Instituto de Psicología Social. Montevideo. Ficha de circulación interna Unidad Curricular de Psicología Social.

LEÓN, A. (S/F). Guía múltiple de la autogestión: un paseo por diferentes hilos de análisis. Material inédito cedido por su autor.

LOURAU, R. (2007). El análisis institucional. Buenos Aires: Amorrortu.

MANER0, R. (1990). Introducción al análisis institucional. Tramas, 121-157. http://www.srmcursos.com/pdf/biblio_psicologia/manero_brito.pdf.

MARQUÉS, J. (2011). Proyecto Profesor Titular Grado 5 para el Instituto de Psicología Social. Montevideo. Ficha de circulación interna Unidad Curricular de Psicología Social.

(2009). Los procesos cooperativos en el desarrollo 
rural sustentable la experiencia de la sociedad de fomento rural La casilla. Tesis de Maestría. Montevideo, Uruguay: Universidad de la República, Facultad de Agronomía.

(2011). Proyecto Profesor Titular Grado 5 para el Instituto de Psicología Social. Montevideo. Ficha de circulación interna Unidad Curricular de Psicología Social.

MARQUÉS, J.; CASTRO, D.; DABEZIES, M. E ISOLA, G. (2005). Los procesos colectivos en cooperativismo y asociativismo. Montevideo: Proyecto Elaboración de Material Pedagógico para las Américas, Red Unircoop.

MARTí, J.P.; THUL F. Y CANCELA, V. (2013). Las empresas recuperadas como cooperativas de trabajo en Uruguay: entre la crisis y la oportunidad. Inédito.

MENDY, M. (2010). Empresas recuperadas por sus trabajadores (ERT) Formas de hacer, formas de ser. En AAW (2010) Gestión Obrera. Del fragmento a la acción colectiva (pp. 31-45). Montevideo: Nordan, Extensión Libros.

MOLAS, A. (1999). Del enfoque bio-psico-social a la concepción social-histórica de las producciones subjetivas. En Fernández, J. y Protesoni, A. (comps). (2001). Psicología Social: Subjetividad y Procesos Sociales. Montevideo: Trapiche.

MORENO, J.L. (1993). Psicodrama. Buenos Aires: Lumen.

NOVAES, A. Y SARDÁ DE FARIAS, M. (2009). ¿Para dónde van las Empresas recuperadas por los Trabajadores brasileños? En Ruggieri, A.; Novaes, A.H.T. y Sardá de Farias,M. (comps.) Crisis y autogestión en el siglo XXI (pp. 75-90). Buenos Aires: Continente.

PAVLOVSKY, E., BOUQUET, C. Y MOCCIO, F. (1991). Psicodrama. Cuando y por qué dramatizar. Buenos Aires: Fundamentos.

PAVLOVSKY, E., KESSELMAN, H. Y FRYDLEWSKY, L. (2007). Las escenas temidas del coordinador de grupos. Buenos Aires: Galerna. 
PERCIA, M. (2009). Notas para pensar lo Grupal. Buenos Aires: Lugar Editorial.

(2011). Inconformidad. Buenos Aires: La Cebra.

(2017). Estancias en común. Buenos Aires: La Cebra.

PICHON RIVIÈRE, E. (1982). El proceso grupal. Del psicoanálisis al Psicología Social. Buenos Aires: Nueva Visión.

RIEIRO, A. (2016). Gestión colectiva en producción. Relaciones sociales a partir de las empresas recuperadas por sus trabajadores en el Cono Sur. Tesis de doctorado. Montevideo, Uruguay: Universidad de la República, Facultad de Ciencias Sociales.

RUGGIERI, A. (2005). Las empresas recuperadas en la Argentina: informe del segundo relevamiento del programa. Buenos Aires: Facultad de Filosofía y Letras.

SARACHU, G. (2009). Limites y posibilidades de la economía social y solidaria: la recuperación del sentido del trabajo en movimiento. Revista Académica PROCOAS-AUGM, 89-105.

(2012). Poder hacer autogestión: Desafios y rupturas necesarias desde las experiencias asociativas populares. En Acosta, Y.; Falero, A.; Rodríguez, A.; Sans,I. y Sarachu,G. (coords.) (2012). Pensamiento crítico en América Latina y sujetos colectivos: debates latinoamericanos(s/p). Montevideo: Trilce.

TARROW, S. (1994). El poder en movimiento. Los movimientos sociales, la acción colectiva y la política. Madrid: Alianza editorial.

TELES, A. (2010). Política afectiva. Apuntes para pensar la vida comunitaria. Entre Ríos: Fundación la Hendija.

TOMMASINO, N. (2017). Pensar en movimiento. Problematizar la vida cotidiana de la autogestión en una empresa recuperada por sus trabajadores. Tesis para optar al título de Magister en Psicología Social, Facultad de Psicología, Montevideo, Udelar.

WEISZ, C. (2012). Obstáculos y facilitadores psico-socio-sim- 
De Prácticas y discursos

Universidad Nacional del Nordeste

Centro de Estudios Sociales

Año 7, Número 10, 2018, Octubre

ISSN 2250-6942
ECONOMÍA SOCIAL Y SOLIDARIA EN MOVIMIENTO: DIÁLOGOS Y APORTES DESDE UNA PSICOLOGÍA SOCIAL PLURAL

SOCIAL AND SOLIDARITY ECONOMY IN MOTION: DIALOGUES AND CONTRIBUTIONS FROM A PLURAL SOCIAL PSYCHOLOGY

bólicos en las ocupaciones autogeneradas y autogestionadas colectivamente. Estudios de casos del Programa Incubadora de Emprendimientos Asociativos Populares (INCOOP-UEC-CSEAM). Tesis de Maestría. Montevideo, Uruguay: Universidad de la República, Facultad de Ciencias Sociales.

WEISZ, C.; RIEIRO, A. Y TOMMASINO, N. (2018). Epistemologías "otras" para las economías alternativas en Uruguay. Universidad de la República (inédito).

ZUFFIAURRE, L. (2002). Hacia una política de los encuentros. En Fernández, J. y Protesonil, A. (eds.) Psicología Social: subjetividad y procesos sociales (pp. 36-41). Montevideo: Psicolibros. 